\title{
Foreword to the Special Issue on Nanofabrication, Atomic and Close-to-atomic Scale Manufacturing
}

\author{
Xichun Luo ${ }^{1}$ Jiang Guo ${ }^{2} \cdot$ Saeed Chavoshi ${ }^{3}$
}

Published online: 15 September 2020

(c) International Society for Nanomanufacturing and Tianjin University and Springer Nature Singapore Pte Ltd. 2020

Nanomanufacturing covers a wide range of manufacturing technologies for enabling nanoscale materials, structures, components, devices, and systems through the manipulation and control of matter at the nano-scale. This is pivotal to our economy, as nanoproducts such as nanomaterials, nanooptics, nanosensors, nanoelectronics, NEMS, etc., are becoming established in major areas of our daily lives and can already be found across a broad spectrum of application areas, especially in sectors such as autos, appliances, electronics, photonics, food, healthcare, and fitness.

At present, the continuous rapid shrinking of feature size for nanoproducts has encouraged engineers and designers to seek alternative nanofabrication patterning methods, as conventional photolithography comes with inherent intrinsic resolution limits. In this regard, some promising nanofabrication techniques including extreme ultraviolet lithography, electron beam lithography, and nanoimprint lithography have been proposed as next-generation lithography (NGL), which has the potential to achieve both high-volume production and very high resolution.

Meanwhile, some subtractive ultra-precision machining and high energy beam machining technologies, additive manufacturing technologies such as atomic layer deposition, and direct self-assembly and transformative manufacturing technologies such as STM atomic manipulation and hydrogen-resist lithography, etc., are striving to achieve atomic-scale precision but also to remove, add, or transform

Xichun Luo

xichun.luo@strath.ac.uk

Jiang Guo

guojiang@dlut.edu.cn

Saeed Chavoshi

s.zare@imperial.ac.uk

1 Centre for Precision Manufacturing, DMEM, University of Strathclyde, Glasgow G1 1XQ, UK

2 Dalian University of Technology, Dalian, China

3 University of Bristol, Bristol BS8 1TH, UK advanced materials at the atomic and close-to-atomic scale, i.e., towards atomic and close-to-atomic scale manufacturing (ACSM).

This special issue of Nanomanufacturing and Metrology, entitled "Nanofabrication, Atomic and Close-to-atomic Scale Manufacturing", collects four original research papers, one communication, and one review article that update the latest developments in nanofabrication and ACSM including a critical review of the state-of-the-art atomic layer removal methods using atomic force microscopy and novel research and development of diamond grooving for high-resolution structural color images, fabrication of PMMA-based micromask sphere for sub-wavelength photolithography, photo-luminescence and Raman spectroscopy study of ionimplanted materials, remote absolute roll-angle measurement, and on-line measurement of ball standards.

We would like to take this opportunity to thank all the contributing authors for their great efforts in presenting their excellent research work for this special issue. Our appreciation also goes to all the reviewing experts who have provided timely invaluable comments and suggestions for the further improvement of the quality of the research papers. The tremendous support from the editorial staff of Nanomanufacturing and Metrology is also highly appreciated.

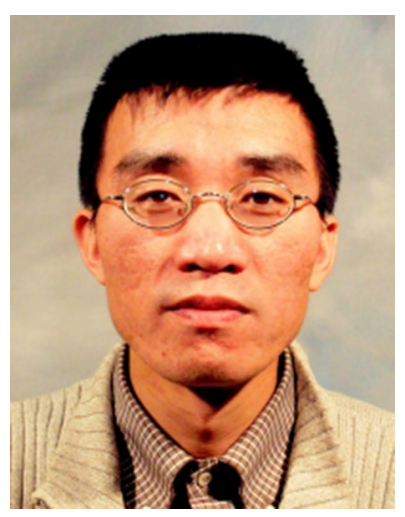

Xichun Luo is a Professor in ultraprecision manufacturing and technical director of Centre for Precision Manufacturing (CPM) at the University of Strathclyde (Glasgow). He is an elected Fellow of the International Society for Nanomanufacturing, the International Association of Advanced Materials, the International Academy of Engineering Technology and an editor for Proceeding of IMechE Part C: Journal of Mechanical Engineering Science, Journal of 
Micromanufacturing and Mechanical Sciences. He also sits in the editorial board for Micromachines, Nanomanufacturing and Metrology. His research has been founded by the EPSRC, EC, Royal Society and Industry. His research interests include ultra-precision machining hybrid micromachining, FIB nanomanufacturing and digital manufacturing, as evidenced by two books and more than 120 papers in peerreviewed highly ranked journals. He chaired two IEEE International Conferences in Automation and Computing in 2014 and 2015. He won UK Institution of Mechanical Engineers (IMechE) 2015 Ludwig Mond Prize for his work in the application of digital technology in micro- and nano-manufacturing.

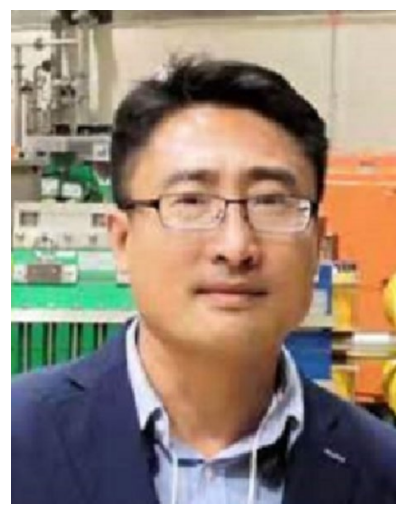

Prof. Jiang Guo is currently a professor at Dalian University of Technology (DUT). He received his Ph.D. from The University of Tokyo in 2013. After graduation, he joined RIKEN as a researcher. In October 2015, he became a scientist in A*STAR (Agency for Science, Technology and Research). He has been actively involved in precision machining related research for over a decade. His research interests include ultraprecision machining, polishing, actuator, mechatronics and neutron optics.

He has published more than 60 journal papers in mechanical engineering and holds several patents. His research work was highlighted in A*STAR Research. He received several funding such as JSPS Grantin-Aid for Scientific Research (B) (2015) and Academic Research
Grant from Mazak Foundation (2014), and awards such as Heidenhain Scholarship from Euspen's 11th International Conference (2011), Young Author's Award for Excellent Presentation from The 20th MAGDA Conference in Pacific Asia (2011) and Best Paper Award from Mazak Foundation (2012). He is a member of European Society for Precision Engineering and Nanotechnology (EUSPEN), American Society for Precision Engineering (ASPE) and Asian Society for Precision Engineering and Nanotechnology (ASPEN). He is the session chair for several conferences such as NanoMan and the reviewer for over 30 SCI journals.

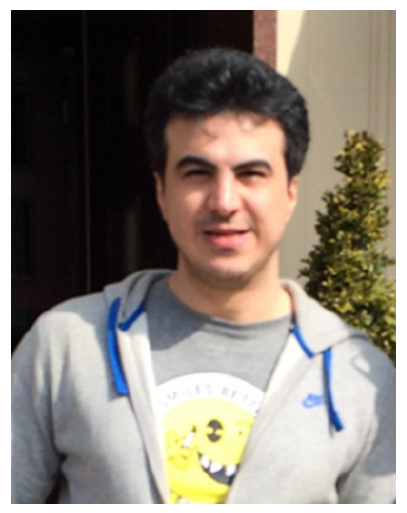

Dr. Saeed Chavoshi is currently a postdoctoral researcher in the Department of Mechanical Engineering at the University of Bristol. Prior to joining Bristol, he was a postdoctoral scholar at Imperial College London, Department of Mechanical Engineering. He received his Ph.D. from the University of Strathclyde in 2016, where he worked in the Centre for Precision Manufacturing. Saeed's research focuses on the multiscale modelling of materials. His remarkable publication list, i.e., 2 book chapters and 33 journal papers (including 2 featured journal papers), with an h-index of 15 and total citation count of 650 , is significantly longer than that of the average young researchers in the field. He has served as reviewers for a number of leading journals in mechanical/ materials engineering. 